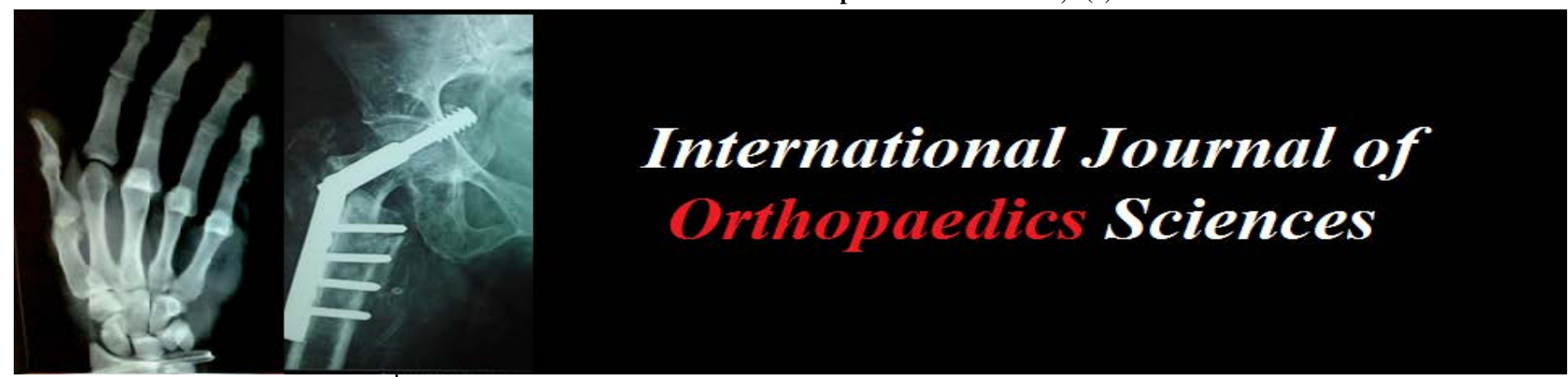

E-ISSN: 2395-1958

P-ISSN: 2706-6630

IJOS 2021; 7(3): 581-586

(C) 2021 IJOS

www.orthopaper.com

Received: 05-05-2021

Accepted: 07-06-2021

Abdulnasser Mohammed Ibrahim Alghamdi

Resident, Department of

Orthopedic, King Fahad

Hospital-Albaha, Saudi Arabia
Corresponding Author:

Abdulnasser Mohammed Ibrahim Alghamdi

Resident, Department of

Orthopedic, King Fahad

Hospital-Albaha, Saudi Arabia

\section{The outcome of arthroscopic surgery in young adults and adults with osteonecrosis: A systematic review and meta-analysis}

\author{
Abdulnasser Mohammed Ibrahim Alghamdi
}

DOI: https://doi.org/10.22271/ortho.2021.v7.i3i.2805

\begin{abstract}
Avascular necrosis of the femoral head affects active young adults between 30-40 years with the risk of progressing to disabling osteoarthritis. Total hip replacement adds more burden to the disease. The role of arthroscopy is to salvage the hip joint at an earlier stage to minimize surgery. The purpose of this review is to explore the efficacy of arthroscopy to salvage hip joints and minimize surgery. A systematic review of the eminent medical databases including PubMed, Embase, Web of Science, and Scopus were searching for the outcome of arthroscopy in cases of avascular necrosis of the femoral head. The extracted data included English literature from 2000 to the current with the age range18 to 70 years. Case studies, general reviews, operative descriptions were excluded. Meta-analysis was performed for the outcomes including age at the surgery, follow-up duration, Harris Hip Score, and conversion to total hip arthroplasty. The random-effect model was used, and heterogeneity was assessed by Tau ${ }^{2}$ and $\mathrm{I}^{2}$ statistics taking $\mathrm{P}$ value less than 0.05 as a significance level. Only four studies out of 173 research results were considered eligible. The mean age of the participants was $36.27 \pm 5.03$ with a follow-up duration of 63.88 \pm 9.68 years. The overall proportion of the operated joints not converted to THA was $0.93 \pm 0.04$ $(P<0.001$; 95\% CI: 0.85-1.00); the proportion of the joints operated for arthroscopic debridement with no conversion to total hip arthroplasty was $0.99 \pm 0.01(P<0.001$; 95\% CI: 0.96-1.02) and the proportion of the joints operated for arthroscopic-assisted core decompression with no conversion to total hip arthroplasty was $0.88 \pm 0.04(P<0.001$; 95\% CI: 0.81- 0.95). Arthroscopy gives successful results in avascular necrosis of the femoral head in Ficat-Arlet stages I and II. However, randomized studies are needed to confirm the results and reach a consensus.
\end{abstract}

Keywords: Avascular necrosis, osteonecrosis, hip replacement, hip arthroplasty, hip joint.

\section{Introduction}

Since the evolvement of flexible fiber-optic scopes for joint diagnosis and treatment two decades ago, the application of arthroscopy on the management of avascular necrosis (AVN) of the femoral head (AVNFH) has been established ${ }^{[1,2]}$. AVN affects young and active adults between 30-40 years of age progressing to disabling osteoarthritis in nearly $80 \%$ of AVN patients ${ }^{[3]}$. Moreover, AVN accounts for 2-12\% of total hip replacement (Maillefert et al., 1996; National Joint Registry (NJR), 2013) adding more burden on active adults ${ }^{[6]}$. The salient role of arthroscopy includes bone conservative surgery for pre-collapse or small post-collapse lesions ${ }^{[7]}$, thus salvaging the hip joint at an earlier stage of AVN and preserve total hip replacement (THR) for the late stages ${ }^{[8]}$. The accumulating evidence from the literature supports the safety and efficacy of arthroscopy as a useful technique in core decompression ${ }^{\text {[9] }}$ and addressing the articular cartilage and labrum lesions of AVN ${ }^{[7]}$.

\section{The Therapeutic Role of Arthroscopy in AVN}

Intra-articular pathology

Intra-articular pathology affecting the hip joints of AVN patients includes labral tear, chondral flaps, and loose bodies (Papavasiliou \& Gliatis, 2017). Labral tear is associated with femoralacetabular impingement, hip dysplasia ${ }^{[10]}$ causing mechanical symptoms such as clicking, locking, giving away, and popping ${ }^{[11]}$. 
The mechanical symptoms affecting patients with AVN joints including locking, buckling, and clicking are associated with chondral flaps and loose bodies ${ }^{[12]}$. AVN patients suffer from pain that accompanies the mechanical symptoms as well ${ }^{[13]}$. Earlier stages of AVN with intra-articular pathology are amenable to arthroscopic surgery with the best results contrary to arthroscopy for advanced stages of AVN with femoral head collapse $[14,16]$. Patients with mechanical symptoms showed better results of arthroscopy than those suffering from joint pains ${ }^{[17]}$. Moreover, it was concluded that arthroscopy is useful to assess and remove intra-articular third bodies as well as joint assessment in case of mysterious symptoms following total hip replacement (McCarthy, 2004; Whitehouse \& Duncan, 2015). Furthermore, arthroscopy is suggested by some others in selected patients of AVN synovitis to relieve pain and improve the range of movement by joint washout and synovectomy (Papavasiliou et al., 2014).

\section{Core decompression}

Core decompression is the salient therapeutic surgery for earlier AVN lesions in the pre-collapse stage. Arthroscopy helps the surgeon to locate the necrotic lesion under vision correctly place the drill tip during fluoroscopic-assisted retrograde drilling procedure ${ }^{[21]}$. Moreover, surgery under vision minimizes the drilling complications of the femoral head such as penetration, over-drilling, and cartilage damage [22, 23]. Furthermore, arthroscopy allows for directing the pin to the middle of the lesion under vision ${ }^{[7]}$. Visualization of the demarcation between the healthy and bleeding bone and the necrotic tissue is an advantage as well ${ }^{[24]}$. Through a small opening at the head-neck junction level, arthroscopy provides a better chance for the convenient placing of the nonvascularized graft, injection of biological material such as fibrin-rich plasma, and removing the necrotic tissue ${ }^{[25]}$ than C-arm fluoroscopy alone ${ }^{[26]}$.

\section{Others}

AVN presents in Legg-calve'-Perthes (LCP) that affect the growing head of the femur between the age of 5 and 8 years [27]. Arthroscopy was proved to be superior to Magnetic Resonant Imaging in detecting intra-articular pathology ${ }^{[12]}$. Arthroscopy in LCP was proved to be safe and efficient in removing the intra-articular loose bodies, labrum tears debridement, cartilage chondroplasty, and osteoplasty of impingement with significant improvement of Harris hip score (HHS) ${ }^{[28]}$.

The application of arthroscopy for the treatment of AVNFH is recent and only a few pieces of literature discussed the subject. Several studies reported the advantages of arthroscopy in AVNFH as regards the reduction of THR and improvement of HHS [9, 29, 31]. Moreover, arthroscopy is preferred over open surgery due to the small incision and minimization of manipulation damage to the articular cartilage as well as its diagnostic role ${ }^{[7,32,33]}$. As the synthesis of the information is lacking, as far as we know, therefore, the purpose of this review is to explore the efficacy of arthroscopy to salvage hip joints in selected cases and to minimize the need for THR in young adults patients with AVNFH.

\section{Materials and Methods}

A literature search was conducted through several medicaloriented databases including PubMed, Embase, Google Schooler, Web of Science, and Scopus. The current research focused on the therapeutic outcome of arthroscopy in cased of AVNFH. The keywords used were 'avascular necrosis', 'osteonecrosis', non-traumatic necrosis' or 'necrosis' and 'arthroscopy', and 'hip joint'. Medical Subject Headings (MeSH) were used to enhance the search process. The search was carried out in 3 steps. The first step was to search on avascular necrosis and related words using wild cards and Boolean operators. The second step was to search on 'arthroscopy' and 'hip joint' together. Finally, we combined the two searches to get the final results.

The inclusion criteria were studied from 2000 to the current, the age range from 18 to 70 years, and English language studies. Case studies, general reviews, operative descriptive studies were excluded as well as editorials and conferencerelated publications. Moreover, the exclusion criteria included traumatic causes of AVN as well. The success rate, the HHS, and the number of THR were evaluated for meta-analysis. The eligible studies for meta-analysis were reviewed for heterogeneity by comparing the outcomes.

\section{Statistical Analysis and Data Synthesis}

The analysis of the continuous and dichotomous outcomes was measured by taking the mean as the point of estimate with a $95 \%$ confidence interval (95\% CI). Meta-analysis was performed for all the outcomes where at least two studies were eligible, and the random effect model was used ${ }^{[34,35] .}$ Heterogeneity was assessed by $\mathrm{Tau}^{2}$ and $\mathrm{I}^{2}$ statistics. Statistical analysis was performed by Open Meta-analyst Cochrane open-source software taking $P<0.05$ as a significance level.

\section{Results \\ Studies Selection}

173 studies were identified through the databases, out of which four studies were examined for eligibility and only five studies were found to meet the inclusion criteria (Figure 1). 


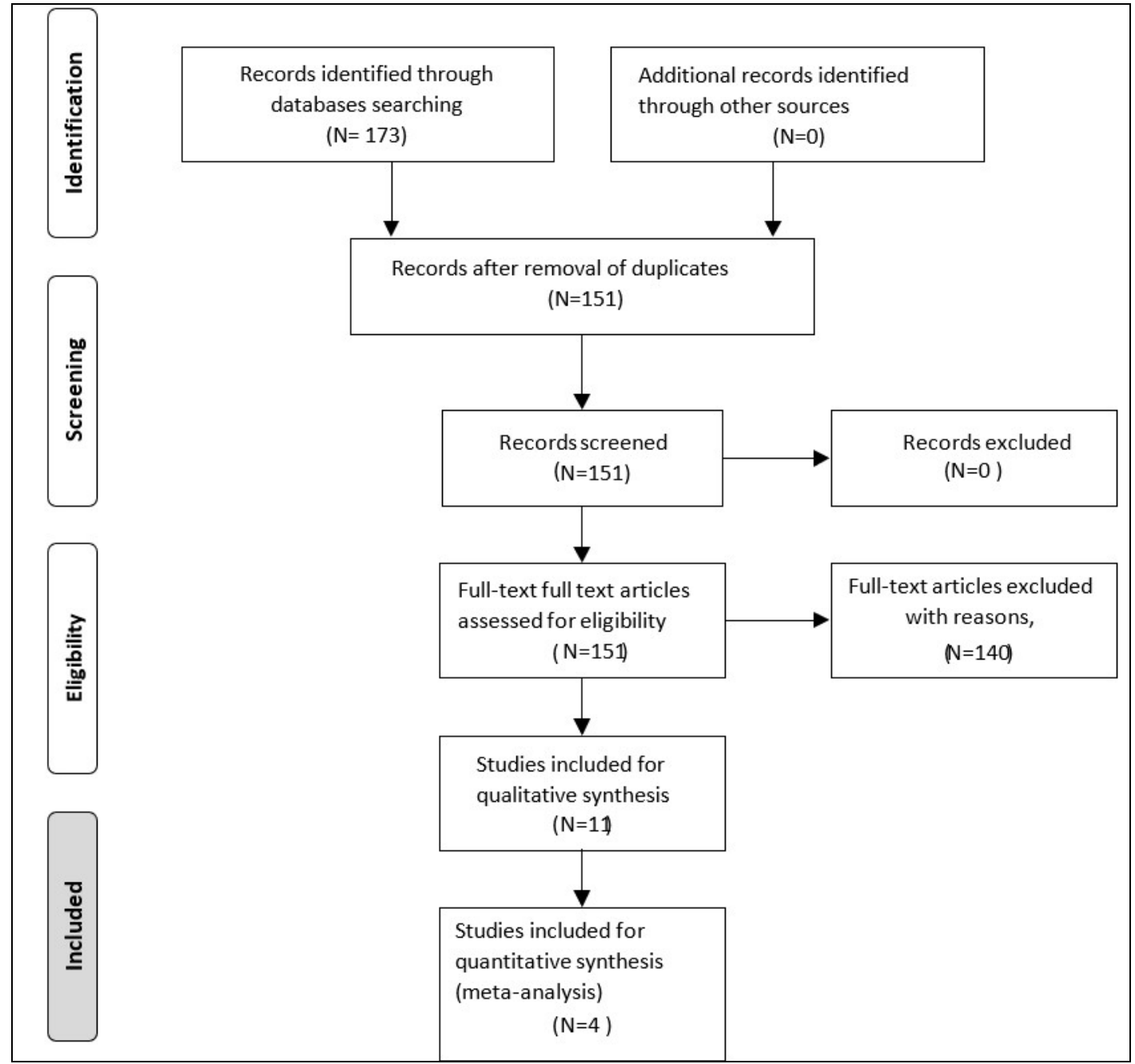

Fig 1: Flow chart for primary studies selection based on PRISMA

\section{Included studies}

Four clinical trial studies were included in this review the characteristics of these studies were described.

\section{Population}

The number of participants in the four studies was 119 patients with non-traumatic AVN. Arthroscopy was carried out for 109 hips with avascular necrosis between 1993 and 2013. The Four studies included 61 males and 58 females aging from 15-84 years with a mean age of $36.2 \pm 2.23$ (95\%CI: 34.01-38.39). The mean follow-up duration was $56.85 \pm 21.28$ (95\% CI:37.00-77.70).

\section{Arthroscopic intervention}

The four studies include two retrospective cohort studies and two case series studies. Arthroscopy was used for the removal of loose bodies and labral tear fixation. Arthroscopy was used to assess core decompression in a pre-collapsed AVN of the femoral head. Arthroscopy for traumatic AVN was excluded.

\section{Outcome}

Age at the surgery. The age outcome was reported as a continuous variable in the four studies with the range (minimum and maximum values) and the mean. Only one study reported the standard deviation, Nazal et al., 2019. The standard deviations for the other studies were recalculated to the value of the pooled standard deviation with a p-value of 0.05 . The mean age of the participants in the four studies was $36.27 \pm 5.03$ ( $P<0.001$, 95\%CI: 26.42-46.13) years (Figure 2 ).

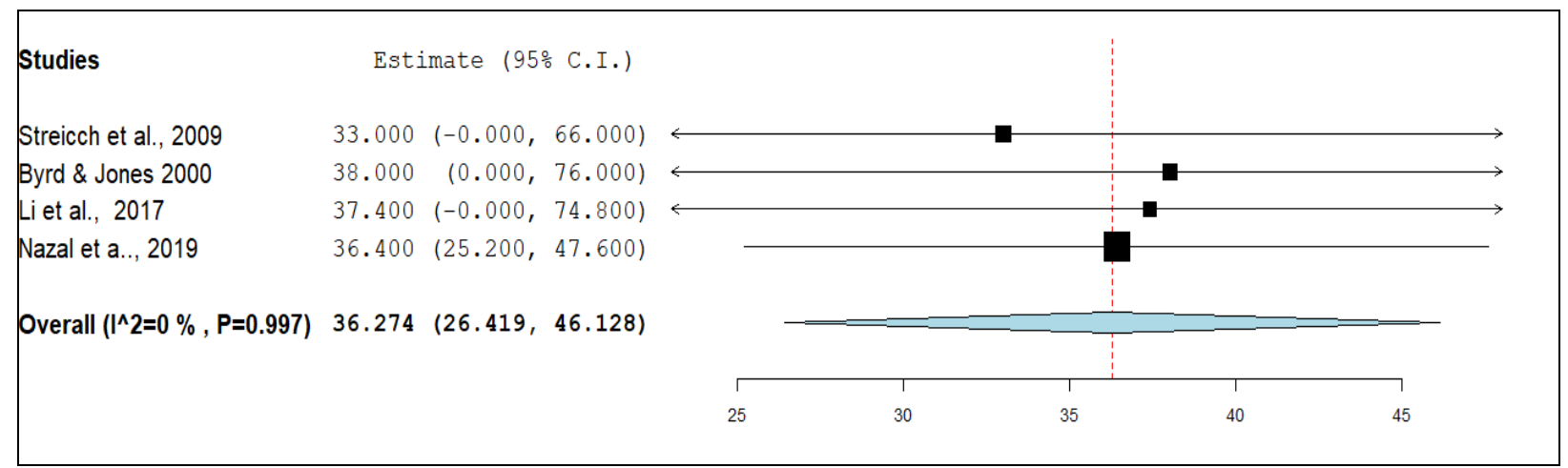

Fig 2: Data synthesis of age outcome 
Duration of follow-up. Data on the follow-up duration after arthroscopy was reported as a continuous variable with the mean and SD in two studies: Li et al., 2017 and Nazal et al., 2019. The standard deviations for the other two studies, Byrd \& Jones 2000 and Streich et al., 2009, were recalculated to the value of the pooled standard deviation with a $\mathrm{p}$-value of
0.05. The analysis of the results of the continuous follow-up duration outcome showed the mean of the follow-up duration equals $63.88 \pm 9.68$ ( $\mathrm{P}<0.001$, 95\% CI: 44.91-82.84) months (Figure 3). Meta-analysis showed a high level of heterogeneity between the studies.

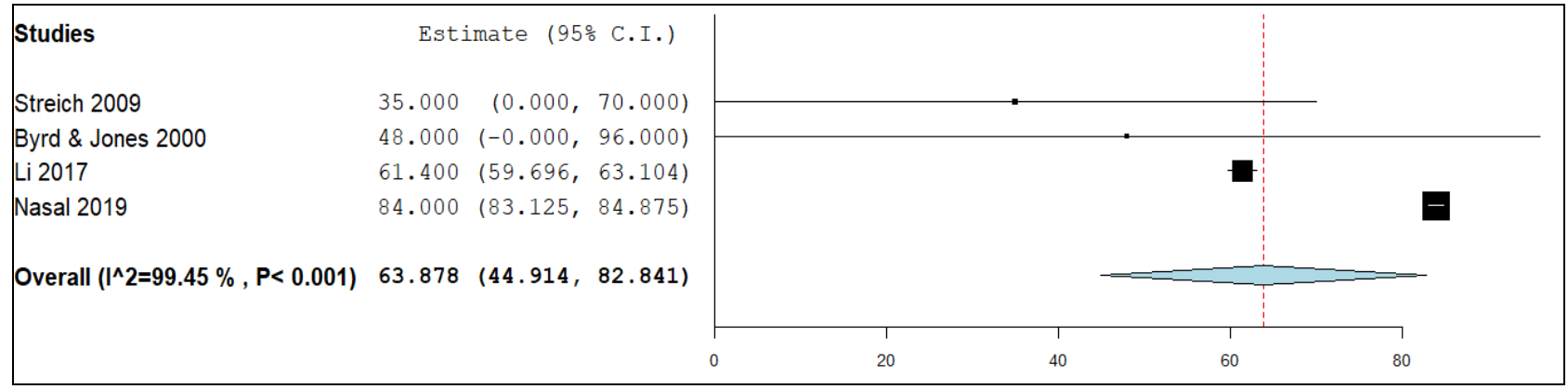

Fig 3: Data analysis of follow-up duration outcome

\section{Harris Hip Score}

Symptoms improvement was measured by HHS score. Symptoms improvement was assessed before and after surgery for the follow-up period stated by the study. One study, Nasal et al., 2019, used Visual Analogue Scale (VAS) for clinical evaluation of the patients. Only the absolute score was mentioned without further analysis in three studies: Streich et al., 2009 and Byrd \& Jones 2000. Meta-analysis was not carried out due to the unavailability of the data.

\section{Conversion to THA}

The conversion to THA was reported as a dichotomous variable. The period to conversion to THA was estimated at the end of the follow-up period. The proportion of joints that had not converted to THA was calculated and used in the statistical analysis and synthesis. Ficat-Alret classification preoperatively was performed before arthroscopy in the four studies. Arthroscopy was performed mainly on stages I and II in the four studies. One study performed arthroscopy on stage IIb ${ }^{[36]}$ and another study included stage III ${ }^{[16]}$ as well. For statistical analysis, the studies were divided into two subgroups including two studies that performed arthroscopy debridement and removal of loose bodies [16, 37] and two studies that performed arthroscopy-assisted core decompression ${ }^{[36,38]}$. The overall proportion of the operated joints not converted to THA was $0.93 \pm 0.04(P<0.001$; 95\% CI: 0.85-1.00); the proportion of the joints operated for arthroscopic debridement with no conversion to THA was $0.99 \pm 0.01(P<0.001 ; 95 \%$ CI: $0.96-1.02$ and the proportion of the joints operated for arthroscopic-assisted core decompression with no conversion to THa was $0.88 \pm 0.04$ $(P<0.001 ; 95 \%$ CI: $0.81-0.95$ (Table 1$)$. The overall heterogeneity was $\mathrm{I}^{2}=66.99 \%$, however, the heterogeneity of the subgroups, debridement and core decompression, was $\mathrm{I}^{2}=$ $0 \%$. (Figure 4).

Table 1: Model results of conversion to THA outcome

\begin{tabular}{|c|c|c|c|c|c|c|c|}
\hline Subgroups & Studies & Estimate & Lower bound & Upper bound & Std. error & p-Val & z-Val \\
\hline Subgroup decompression & 2 & 0.879 & 0.808 & 0.950 & 0.036 & $<0.001$ & 24.248 \\
\hline Subgroup debridement & 2 & 0.988 & 0.961 & 1.015 & 0.014 & $<0.001$ & 72.527 \\
\hline Overall & 4 & 0.926 & 0.849 & 1.002 & 0.039 & $<0.001$ & 23.632 \\
\hline
\end{tabular}

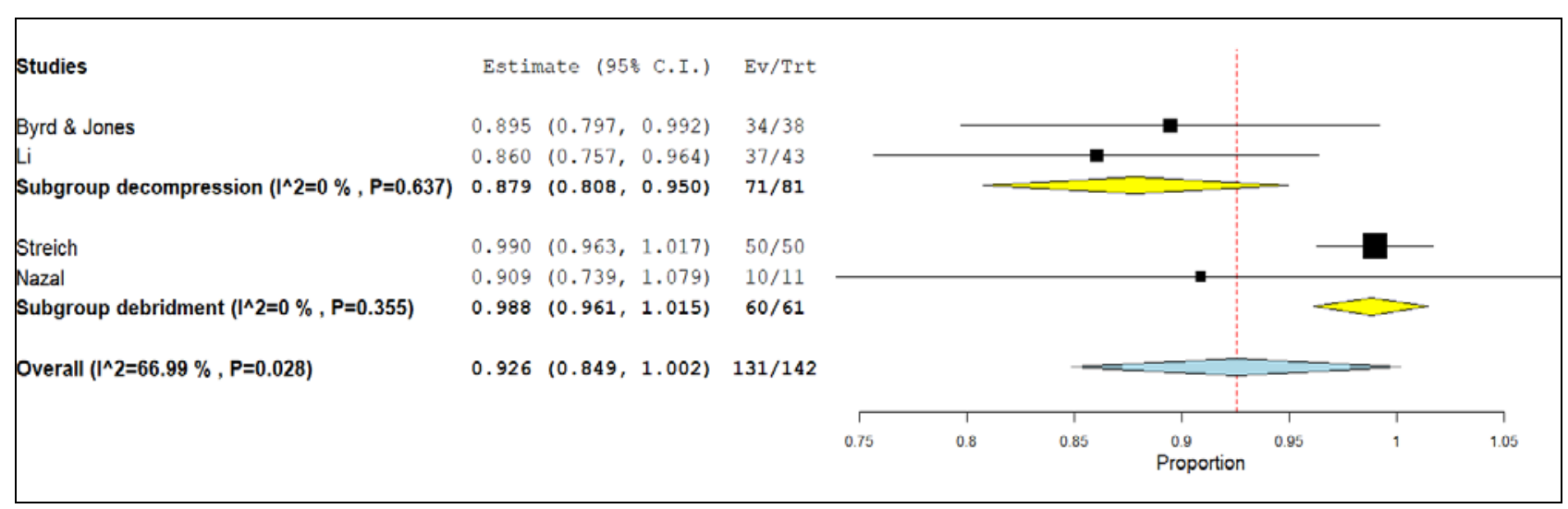

Fig 4: Data analysis of conversion to THA

\section{Discussion}

\section{Summary of Main Results}

The purpose of this review is to analyze and summarize the available data on the efficacy of arthroscopy in the surgical management of the non-traumatic AVN of the hip joint. Four studies conducted between 1993 to 2013 comprehending 119 cases having non-traumatic AVN hip were found eligible for the review. As for the success of the arthroscopy surgery, conversion to THA was assessed in the four studies. The review revealed that arthroscopy was performed mainly for 
either debridement or loose body removal or as an assistant in core decompression surgery with satisfactory results measured by the proportion of conversion to THA. The successful results were higher in grades I and II of the FicatArlet classification. As the age range was consistent in the four studied, the duration of the follow-up showed marked variations across the studies.

\section{Biase and Limitations}

There was a high level of heterogeneity considering postarthroscopy follow-up duration. The high level of heterogeneity reflects the lack of consensus of the follow-up period. The heterogeneity was relatively high when the conversion to THA was considered, however, this heterogeneity disappeared when the studies were sub grouped according to the type of the operation. The limitations that we are aware of include the inclusion of case series and lack of RCT studies. Moreover, the overall high heterogeneity would influence the results of the review as well.

\section{Comparing and Contrasting with Literature Reviews}

Core decompression is a joint preserving surgical procedure to salvage the hip joints in cases of AVN that should be attempted in ARCO (Association Research Circulation Osseous) stage I - III ${ }^{[7]}$. Core decompression in cases of AVN reduces pain, enhances bone regeneration [39]. Moreover, percutaneous drilling of core decompression leads to intramedullary pressure reduction and improvement of the blood flow in the subchondral region that could result in reversal of the necrosis process of the femoral head [25, 40]. Arthroscopies provide safety drilling in three levels without injuring the femoral head or neck [38, 40, 41]. Arthroscopy is useful for necrotic lesions located on the lateral (cranial) posterior and ventral femoral head ${ }^{[7]}$. Arthroscopy reduces exposure to radiation ${ }^{[42]}$. Moreover, diagnosis can be further confirmed and other undetected pathologies could be found (Papavasiliou \& Gliatis, 2017). The result of this review matches the conclusions of the literature in that arthroscopy is effective in assessing core decompression surgery with less conversion to THA when performed earlier in the disease progression.

\section{Implications for Practice and Research}

The available data in this review encourages the use of arthroscopy for the management of AVN of the hip joints. Arthroscopy in the early stages of AVN of the hip can salvage the hip and minimize the need for THA that is crucial in young men. RCT studies are needed to compare the outcome of arthroscopy and conventional procedures. Moreover, RCT studies would provide information about the efficacy and safety of arthroscopy compared with the conventional procedures for core decompression of the hip in cases of AVN. As the follow-up duration showed heterogeneity, it is worth noting that there is a need to standardize the follow-up duration following arthroscopy for better judgment of the outcomes.

\section{References}

1. McCarthy J, Puri L, Barsoum W, Lee JA, Laker M, Cooke P. Articular cartilage changes in avascular necrosis: an arthroscopic evaluation. Clin Orthop Relat Res 2003;(406):64-70.

2. Sekiya JK, Ruch DS, Hunter DM, Pope TL, Koman LA, Poehling GG et al. Hip arthroscopy in staging avascular necrosis of the femoral head. J South Orthop Assoc
2000;9(4):254-61.

3. Hunt LP, Ben-Shlomo Y, Clark EM, Dieppe P, Judge A, MacGregor AJ et al. 90-day mortality after 409096 total hip replacements for osteoarthritis, from the National Joint Registry for England and Wales: a retrospective analysis. The Lancet [Internet] 2013;382(9898):1097104. Available from: https://www.sciencedirect.com/science/article/pii/S01406 73613617493

4. Maillefert JF, Tavernier C, Toubeau M, Brunotte F. Nontraumatic avascular necrosis of the femoral head. J Bone Joint Surg Am 1996;78(3):473-4.

5. National Joint Resgistry (NJR). National Joint Registry 10th Annual Report 2013 [Internet]. HQIP 2013. [cited 2021 May 11]. Available from: https://www.hqip.org.uk/resource/national-joint-registry10th-annual-report-2013/

6. Papavasiliou AV, Gliatis I. Specific Tissues and Conditions: Osteonecrosis, Avascular Necrosis. Hip Joint Restoration [Internet] 2017, 555-9. Available from: https://link.springer.com/chapter/10.1007/978-1-46140694-5_55

7. Theopold J, Armonies S, Pieroh P, Hepp P, Roth A. Nontraumatic avascular necrosis of the femoral head: Arthroscopic and navigation-supported core decompression. Oper Orthop Traumatol 2020;32(2):10715.

8. Delniotis I, Leidinger B. Hip Arthroscopy as Part of a Salvage Procedure for Avascular Necrosis (AVN) in a 7Year-Old Child. Am J Case Rep 2019;20:941-7.

9. Guo HS, Tian YJ, Liu G, An L, Zhou ZG, Liu HZ. [Arthroscopy-guided core decompression and bone grafting combined with selective arterial infusion for treatment of early stage avascular necrosis of femoral head]. Zhongguo Gu Shang 2018;31(1):56-61.

10. Izumida $H$, Kanaji A, Nishiwaki $T$, Shimizu $H$, Fujie A, Tando $\mathrm{T}$ et al. Acetabular labral tear complicating idiopathic osteonecrosis of the femoral head treated by labral repair with hip arthroscopy: a case report. J Med Case Rep [Internet] 2014;8:372. Available from: https://www.ncbi.nlm.nih.gov/pmc/articles/PMC430414/

11. Kamenaga $\mathrm{T}$, Hashimoto S, Hayashi S, Takayama K, Niikura T, Kuroda R, et al. Larger Acetabular Labrum Is Associated With Hip Dysplasia, Joint Incongruence, and Clinical Symptoms. Arthroscopy 2020;36(9):2446-53.

12. Tiwari V, Gamanagatti S, Mittal R, Nag H, Khan SA. Correlation between MRI and hip arthroscopy in children with Legg-Calve-Perthes disease. Musculoskelet Surg 2018;102(2):153-7.

13. Hatanaka H, Motomura G, Ikemura S, Kubo Y, Utsunomiya $\mathrm{T}$, Baba $\mathrm{S}$ et al. Differences in magnetic resonance findings between symptomatic and asymptomatic pre-collapse osteonecrosis of the femoral head. Eur J Radiol 2019;112:1-6.

14. Guo HS, Tian YJ, Liu G, An L, Zhou ZG, Liu HZ. [Arthroscopy-guided core decompression and bone grafting combined with selective arterial infusion for treatment of early stage avascular necrosis of femoral head]. Zhongguo Gu Shang 2018;31(1):56-61.

15. Larson CM, Swaringen J, Morrison G. A review of hip arthroscopy and its role in the management of adult hip pain. Iowa Orthop J 2005;25:172-9.

16. Nazal MR, Parsa A, Martin SD. Mid-term outcomes of arthroscopic-assisted Core decompression of Precollapse osteonecrosis of femoral head-minimum of 5 year follow- 
up. BMC musculoskeletal disorders 2019, 20(1).

17. O’leary JA, Berend K, Vail TP. The relationship between diagnosis and outcome in arthroscopy of the hip. Arthroscopy 2001;17(2):181-8.

18. McCarthy JC. Hip arthroscopy: when it is and when it is not indicated. Instr Course Lect 2004;53:615-21.

19. Whitehouse MR, Duncan CP. Arthroscopy as a diagnostic tool for painful turn-on corrosion after hip arthroplasty. Knee Surg Sports Traumatol Arthrosc 2015;23(9):2601-4.

20. Papavasiliou A, Yercan HS, Koukoulias N. The role of hip arthroscopy in the management of osteonecrosis. J Hip Preserv Surg 2014;1(2):56-61.

21. Smart LR, Oetgen M, Noonan B, Medvecky M. Beginning hip arthroscopy: indications, positioning, portals, basic techniques, and complications. Arthroscopy 2007;23(12):1348-53.

22. Dienst M. [Hip arthroscopy. Technique for positioning and distraction]. Orthopade 2006;35(1):33-40.

23. Kuhns BD, Frank RM, Pulido L. Open and Arthroscopic Surgical Treatment of Femoroacetabular Impingement. Front Surg 2015;2:63.

24. Govaers K, Meermans G, Bortier H, Londers J. Endoscopically assisted core decompression in avascular necrosis of the femoral head. Acta Orthop Belg 2009;75(5):631-6.

25. Guadilla J, Fiz N, Andia I, Sánchez M. Arthroscopic management and platelet-rich plasma therapy for avascular necrosis of the hip. Knee Surg Sports Traumatol Arthrosc 2012;20(2):393-8.

26. Rocchi M, Del Piccolo N, Mazzotta A, Giavaresi G, Fini $\mathrm{M}$, Facchini $\mathrm{F}$ et al. Core decompression with bone chips allograft in combination with fibrin platelet-rich plasma and concentrated autologous mesenchymal stromal cells, isolated from bone marrow: results for the treatment of avascular necrosis of the femoral head after 2 years minimum follow-up. Hip Int 2020;30(2_suppl):3-12.

27. Leroux J, Abu Amara S, Lechevallier J. Legg-CalvéPerthes disease. Orthopaedics \& Traumatology: Surgery \& Research [Internet] 2018;104(1, Supplement):S10712. Available from: https://www.sciencedirect.com/science/article/pii/S18770 56817303249

28. Goyal T, Barik S, Gupta T. Hip Arthroscopy for Sequelae of Legg-Calve-Perthes Disease: A Systematic Review. Hip Pelvis 2021;33(1):3-10.

29. Beck DM, Park BK, Youm T, Wolfson TS. Arthroscopic Treatment of Labral Tears and Concurrent Avascular Necrosis of the Femoral Head in Young Adults. Arthrosc Tech [Internet] 2013;2(4):e367-71. Available from: https://www.ncbi.nlm.nih.gov/pmc/articles/PMC388271/

30. Economopoulos DG, Triantafyllopoulos IK. Minimally invasive treatment of femoral head avascular necrosis in a beta thalassemia carrier. A case-report. J Musculoskelet Neuronal Interact [Internet]. 2019;19(3):374-8. Available from: https://www.ncbi.nlm.nih.gov/pmc/articles/PMC673756/

31. Lim C, Cho TJ, Shin CH, Choi IH, Yoo WJ. Functional Outcomes of Hip Arthroscopy for Pediatric and Adolescent Hip Disorders. Clin Orthop Surg. 2020;12(1):94-9.

32. Andronic $\mathrm{O}$, Weiss $\mathrm{O}$, Shoman $\mathrm{H}$, Kriechling $\mathrm{P}$, Khanduja V. What are the outcomes of core decompression without augmentation in patients with nontraumatic osteonecrosis of the femoral head? Int
Orthop 2021;45(3):605-13.

33. Lim C, Cho TJ, Shin CH, Choi IH, Yoo WJ. Functional Outcomes of Hip Arthroscopy for Pediatric and Adolescent Hip Disorders. Clin Orthop Surg 2020;12(1):94-9.

34. Viechtbauer W. Conducting meta-analyses in $\mathrm{R}$ with the metafor package. Journal of statistical software 2010;36(3):1-48.

35. Wallace BC, Dahabreh IJ, Trikalinos TA, Lau J, Trow P, Schmid $\mathrm{CH}$. Closing the gap between methodologists and end-users: R as a computational back-end. J Stat Softw 2012;49(5):1-15.

36. Byrd JW, Jones KS. Prospective analysis of hip arthroscopy with 2-year follow-up. Arthroscopy 2000;16(6):578-87.

37. Streich NA, Gotterbarm T, Barié A, Schmitt H. Prognostic value of chondral defects on the outcome after arthroscopic treatment of acetabular labral tears. Knee Surg Sports Traumatol Arthrosc 2009;17(10):1257-63.

38. Li J, Li Z, Su X, Liu C, Zhang $\mathrm{H}$, Wang $\mathrm{K}$. [Effectiveness of multiple small-diameter drilling decompression combined with hip arthroscopy for early osteonecrosis of the femoral head]. Zhongguo Xiu Fu Chong Jian Wai Ke Za Zhi 2017;31(9):1025-30.

39. Andriolo L, Merli G, Tobar C, Altamura SA, Kon E, Filardo G. Regenerative therapies increase survivorship of avascular necrosis of the femoral head: a systematic review and meta-analysis. Int Orthop 2018;42(7):1689704.

40. Wang W, Liu L, Dang X, Ma S, Zhang M, Wang K. The effect of core decompression on local expression of BMP-2, PPAR- $\gamma$ and bone regeneration in the steroidinduced femoral head osteonecrosis. BMC Musculoskelet Disord 2012;13:142.

41. Wang W, Hu W, Yang P, Dang XQ, Li XH, Wang KZ. Patient-specific core decompression surgery for earlystage ischemic necrosis of the femoral head. PLoS One 2017;12(5):e0175366.

42. Beckmann J, Goetz J, Baethis H, Kalteis T, Grifka J, Perlick L. Precision of computer-assisted core decompression drilling of the femoral head. Arch Orthop Trauma Surg 2006;126(6):374-9. 